ISAHP 2001, Berne, Switzerland, August 2-4, 2001

\title{
STABILITY OF DISTRIBUTIVE AND IDEAL AHP MODELS WITH RESPECT TO ADDITION OF A NEW ALTERNATIVE
}

\author{
A. Ekárt; S.Z. Németh \\ Computer and Automation Research Institute \\ Hungarian Academy of Sciences \\ P.O.B. 63, H-1518 Budapest-Hungary \\ e-mail: \{ekart, snemeth\}@sztaki.hu, URL: www.sztaki.hu/ \{ekart, snemeth\}
}

Keywords: Decision support systems, Multiple criteria analysis, AHP

Summary: Stability of the order of alternatives is studied in distributive and ideal AHP models when a new alternative is added. We developed a method for finding the maximal box within which the new alternative can take values, so that the order of the initial alternatives remains unchanged. The method is demonstrated on the well-known Expert Choice problem of finding the best retail site for an icecream outlet.

\section{Introduction}

In the distributive (ideal) AHP model $n$ alternatives $A_{1}, A_{2}, \ldots, A_{n}$ are ranked by using $m$ criteria $C_{1}, C_{2}, \ldots, C_{m}$. If $w_{i} \geq 0$ is the weight of the $i$-th criterion $\left(\sum_{l=1}^{m} w_{l} \neq 0\right)$ and $a_{i j}>0$ the value of the $j$-th alternative on the $i$-th criterion, $i \in\{1,2, \ldots, m\} ; j \in\{1,2, \ldots, n\}$, then the alternatives are ordered by their aggregate values

$$
x_{j}=\sum_{i=1}^{m} \hat{w}_{i} \hat{a}_{i j}, j=1,2, \ldots, n,
$$

where $\hat{w}_{i}=w_{i} / \sum_{l=1}^{m} w_{l}$ and $\hat{a}_{i j}=a_{i j} / \sum_{k=1}^{n} a_{i k} \quad\left(\hat{a}_{i j}=a_{i j} / \max \left\{a_{i k}: k=1, \ldots, n\right\}\right)$. These models use a relative measurement method and contrarily to the ratings model, it is possible to have reversal in the rank of alternatives if a new alternative is added or another one deleted. Examples for rank reversal can be found in (Saaty, 1986) and (Saaty, 1987). The structure of possible rank reversals was studied in details by Saaty (Saaty, 1987).

In the Tutorial of the "Expert Choice 2000" multicriteria decision software it is mentioned that when using the distributive or ideal AHP model, the comparison of alternatives with different order of magnitude should be avoided. This could cause severe rank reversals. S. Z. Németh investigated in (Németh, 2000) how strong the influence of a new alternative on the order of previous ones can be.

We shall analyze how stable is the order of the alternatives with respect to the addition of a new alternative in distributive (ideal) AHP models. We shall introduce a global stability index, which measures the influence of a new alternative on the order of the original alternatives. 


\section{Main results}

Consider an arbitrary multicriteria decision problem with $n$ alternatives

$$
A_{1}, A_{2}, \ldots, A_{n}
$$

and $m$ criteria

$$
C_{1}, C_{2}, \ldots, C_{m}
$$

Denote by $w_{1}, w_{2}, \ldots, w_{m}$ the weights of criteria $C_{1}, C_{2}, \ldots, C_{m}$, respectively.

Consider a new alternative $Z$. Let $z_{i}>0$ be the value of $Z$ on the $i$-th criterion. The new aggregated values will be

$$
x_{j}^{\prime}=\sum_{i=1}^{m} \hat{w}_{i} \hat{a}_{i j}^{\prime}, \quad j=1,2, \ldots, n,
$$

where $\hat{a}_{i j}^{\prime}=a_{i j} /\left(z_{i}+\sum_{k=1}^{n} a_{i k}\right)\left(\hat{a}_{i j}^{\prime}=a_{i j} / \max \left\{z_{i}, \max \left\{a_{i k}: k=1, \ldots, n\right\}\right\}\right)$. Hence we have

$$
\hat{a}_{i j}^{\prime}=\phi_{i}\left(z_{i}\right) a_{i j},
$$

where $\phi_{i}: t \mapsto 1 /\left(t+\sum_{k=1}^{n} a_{i k}\right)\left(\phi_{i}: t \mapsto 1 / \max \left\{t, \max \left\{a_{i k}: k=1,2, \ldots, n\right\}\right\}\right)$ is a monotone decreasing function of $t>0$ such that

$$
\lim _{t \rightarrow+\infty} \phi_{i}(t)=0
$$

By using (1) the new aggregated values will be

$$
x_{j}^{\prime}=\sum_{i=1}^{m} b_{i j} \phi_{i}\left(z_{i}\right), j=1,2, \ldots, n,
$$

where $b_{i j}=\hat{w}_{i} a_{i j}$. There is a permutation $\tau$ of the set $\{1,2, \ldots, n\}$, so that to have $x_{\tau(1)} \geq x_{\tau(2)} \geq \ldots \geq x_{\tau(n)}$.

Denote by $D(E)$ the artificially created "minimal" ("maximal") alternative of components $d_{i}=\min \left\{a_{i j}: j=1,2, \ldots, n\right\}\left(e_{i}=\max \left\{a_{i j}: j=1,2, \ldots, n\right\}\right)$.

Definition 1 We say that the ratio between the order of magnitudes of alternative $Z$ and alternatives $A_{j}$, $j=1,2, \ldots, n$ is $\lambda\left(\lambda \in\left[\sqrt{\max \left\{d_{i} / e_{i}: i=1,2, \ldots, n\right\}},+\infty\right)\right)$ if $z_{i} \in\left[d_{i} / \lambda, e_{i} \lambda\right], i=1,2, \ldots, m$ and there is an $i_{0} \in\{1,2, \ldots, m\}$ such that $z_{i_{0}} \in\left\{d_{i_{0}} / \lambda, e_{i_{0}} \lambda\right\}$. 
Definition 2 We call the stability index ${ }^{I}$ with respect to the order $x_{\tau(j)} \geq x_{\tau(j+1)}, j \in\{1,2, \ldots, n-1\}$ the maximal ratio $\alpha_{j}$ between the order of magnitudes of the alternative $Z$ and alternatives $A_{k}, k=1,2, \ldots, n$ so that $x_{\tau(j)}^{\prime} \geq x_{\tau(j+1)}^{\prime}$. In other words if $z_{i} \in\left\lfloor d_{i} / \alpha_{j}, e_{i} \alpha_{j}\right\rfloor, i=1,2, \ldots, m$, then $x_{\tau(j)}^{\prime} \geq x_{\tau(j+1)}^{\prime}$ and $\alpha_{j}$ is maximal with respect to this property.

Definition $3 \alpha=\min \left\{\alpha_{j}: j=1,2, \ldots, n\right\}$ is called the global stability. It is the maximal ratio between the order of magnitudes of the alternative $Z$ and alternatives, $A_{j}, j=1,2, \ldots, n$ so that the order of the alternatives remains unchanged.

Our aim is to calculate the global stability $\alpha$. By using (3) we have

$$
x_{\tau(j)}^{\prime}-x_{\tau(j+1)}^{\prime}=\sum_{i=1}^{m} f_{i, \tau(j)} \phi_{i}\left(z_{i}\right)
$$

where $f_{i j}=b_{i j}-b_{i, j+1}$. Let

$$
P_{j}=\left\{i \in\{1,2, \ldots, m\}: f_{i, \tau(j)} \geq 0\right\}=\left\{i \in\{1,2, \ldots, m\}: a_{i, \tau(j)} \geq a_{i, \tau(j+1)}\right\}
$$

and

$$
N_{j}=\left\{i \in\{1,2, \ldots, m\}: f_{i, \tau(j)}<0\right\}=\left\{i \in\{1,2, \ldots, m\}: a_{i, \tau(j)}<a_{i, \tau(j+1)}\right\} .
$$

In order to obtain the global stability we must calculate $\alpha_{j}, j=1,2, \ldots, n$. It is easy to see that

$$
\alpha_{j}=\max \left\{\lambda \in I: \min \left\{x_{\tau(j)}^{\prime}-x_{\tau(j+1)}: z_{i} \in I_{\lambda}^{i}, i=1,2, \ldots, m\right\}\right\} \geq 0,
$$

where $I=\left[\sqrt{\max \left\{d_{i} / e_{i}: i=1,2, \ldots, m\right\}},+\infty\right)$ and $I_{\lambda}^{i}=\left[d_{i} / \lambda, e_{i} \lambda\right]$.

Since $\phi_{i}$ is monotone and $f_{i j}=\left(w_{i} / \sum_{l=1}^{m} w_{l}\right)\left(a_{i j}-a_{i, j+1}\right)$ (4) yields:

Theorem 1 The following relations hold:

$$
\alpha_{j}=\max \left\{\lambda \in I: \sum_{i \in P_{j}} w_{i}\left(a_{i, \tau(j)}-a_{i, \tau(j+1)}\right) \phi_{I}\left(e_{i} \lambda\right)+\sum_{i \in P_{j}} w_{i}\left(a_{i, \tau(j)}-a_{i, \tau(j+1)}\right) \phi_{i}\left(\frac{d_{i}}{\lambda}\right) \geq 0\right\}
$$

and

$$
\alpha=\min \left\{\alpha_{j}: j=1,2, \ldots, n\right\}
$$

For $j=1,2, \ldots, n$ we define the function $\psi_{j}: I \rightarrow \mathbf{R}$ by

$$
\psi_{j}(\lambda)=\sum_{i \in P_{j}} w_{i}\left(a_{i, \tau(j)}-a_{i, \tau(j+1)}\right) \phi_{I}\left(e_{i} \lambda\right)+\sum_{i \in P_{j}} w_{i}\left(a_{i, \tau(j)}-a_{i, \tau(j+1)}\right) \phi_{i}\left(\frac{d_{i}}{\lambda}\right) .
$$

\footnotetext{
${ }^{1}$ A similar stability index for weights was introduced in (Mészáros and Rapcsák, 1996).
} 
It is easy to see that $\psi_{j}$ is decreasing and by using (2) $\lim _{\lambda \rightarrow+\infty} \psi_{j}(\lambda) \leq 0$ for all $j=1,2, \ldots, n$. If $\psi_{j}\left(\sqrt{\max \left\{d_{i} / e_{i}: i=1,2, \ldots, m\right\}}\right)<0$, then by convention we put $\alpha_{j}=-\infty$ (since the supreme of an empty set is $-\infty)$. In this case we have $\alpha=-\infty$. Using this convention we have:

Theorem 2 If $\psi_{j}\left(\sqrt{\max \left\{d_{i} / e_{i}: i=1,2, \ldots, m\right\}}\right) \geq 0$, then $\alpha_{j}$ is the smallest root ${ }^{2}$ of the equation

$$
\sum_{i \in P_{j}} w_{i}\left(a_{i, \tau(j)}-a_{i, \tau(j+1)}\right) \phi_{i}\left(e_{i} \lambda\right)+\sum_{i \in P_{j}} w_{i}\left(a_{i, \tau(j)}-a_{i, \tau(j+1)}\right) \phi_{i}\left(\frac{d_{i}}{\lambda}\right)=0 .
$$

If $\psi_{j}\left(\sqrt{\max \left\{d_{i} / e_{i}: i=1,2, \ldots, m\right\}}\right)<0$, then $\alpha_{j}=-\infty$.

Proof. Since $\lim _{\lambda \rightarrow+\infty} \psi_{j}(\lambda) \leq 0$, if $\psi_{j}\left(\sqrt{\max \left\{d_{i} / e_{i}: i=1,2, \ldots, m\right\}}\right) \geq 0$, then by the Cauchy theorem for continuous functions equation (5) must have a root. Since $\psi_{j}$ is decreasing, $\alpha_{j}$ is the smallest root of equation (5).

Remark If $a_{i, \tau(j)} \geq a_{i, \tau(j+1)}$ for all $i=1,2, \ldots, m$, i.e., the alternative $A_{\tau(j)}$ Pareto dominates the alternative $A_{\tau(j+1)}$, then $\alpha_{j}=+\infty$. This means that the order of alternatives $A_{\tau(j)}, A_{\tau(j+1)}$ remains unchanged if any new alternative is added. If the alternatives $A_{k}, k=1,2, \ldots, n$ are Pareto ordered, then $\alpha=+\infty$. This means that the order of alternatives $A_{k}, k=1^{\prime}, 2, \ldots, n$ remains unchanged if a new alternative is added.

Example Consider the example problem of Expert Choice, which consists of selecting the best retail site for a new icecream outlet.

The selection is based on the following criteria:

- $C_{1}=$ Visibility of site location,

- $C_{2}=$ The number of competitors in the area

- $C_{3}=$ The number of passerby who could be clients

- $C_{4}=$ Monthly rental cost per square foot.

The possible alternatives are the following sites:

- $A_{1}=$ Suburban Center

- $A_{2}=$ Suburban Mall Location

- $A_{3}=$ Main Street Business Location.

After pairwise comparing the importance of the criteria and the preferences of alternatives with respect to the criteria, the following table (containing the weights of criteria and the values of the alternatives with respect to the criteria) is obtained:

\footnotetext{
${ }^{2}$ If $\lim _{\lambda \rightarrow+\infty} \psi_{j}(\lambda)=0$, then by convention we take the root of equation (5) to be $+\infty$.
} 


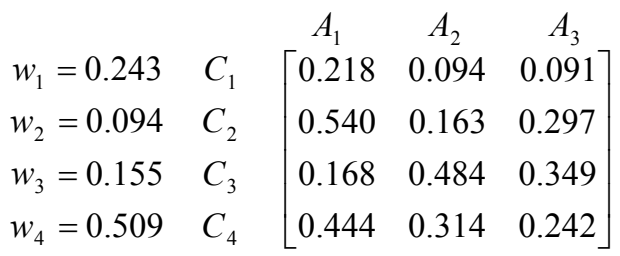

The columns of the matrix will be identified with the corresponding alternatives.

For the computation of the global stability, we made a MATLAB program. Our program also calculates the maximal box where a new alternative can take values from, so that the order of the original alternatives is preserved. For our example, in the distributive (ideal) case the stability is 1.385 (1.246) and the maximal box is

$$
\begin{aligned}
& {[0.066,0.957] \times[0.118,0.748] \times[0.121,0.670] \times[0.175,0.615]} \\
& ([0.066,0.957] \times[0.118,0.748] \times[0.121,0.670] \times[0.175,0.615]) .
\end{aligned}
$$

If a new alternative is chosen from this box then the order of alternatives $A_{1}, A_{2}, A_{3}$ (given by the relations $x_{1} \geq x_{2} \geq x_{3}$ ) remains unchanged and the box is maximal with respect to this property.

\section{Acknowledgments}

This work was supported by grant No. T029572 of the National Research Foundation of Hungary. S. Z. Németh was supported by the Bolyai János Research Fellowship. The authors are grateful to Csaba Mészáros and János Fülöp for many helpful discussions.

\section{References}

Belton, V. (1986), "A comparison of the analytic hierarchy process and a simple multi-attribute value function", European Journal of Operational Research, 26, 7-21.

Mészáros, Cs. - Rapcsák, T. (1996), "On sensitivity analysis for a class of decision systems, Decision Support Systems, 16 (3), 231-240.

Németh, S. Z. (2000) "Domination of one criterion in distributive AHP modes by introducing a new alternative", Working paper of the Laboratory of Operations Research and Decision Systems (LORDS), WP 2000-11, Budapest, MTA SZTAKI, 7 p.

Saaty, T. L. (1986), "Absolute and relative measurement with the AHP. The most livable cities in the United States", Socio-Econ. Plann. Sci., 20 (6), 327-331.

Saaty, T. L. (1987), "Rank generation, preservation and reversal in the analytic hierarchy decision process", Decision Sciences, 18 (2), Spring. 
